\title{
Clinical and Cost Consequences of Metabolic Effects of Lurasidone Versus Other Atypical Antipsychotics in Schizophrenia
}

\author{
Krithika Rajagopalan ${ }^{1}$, Shien Guo ${ }^{*}, 2$ Luis Hernandez ${ }^{2}$, Julia Green ${ }^{2}$ and Antony Loebel ${ }^{1}$ \\ ${ }^{I}$ Sunovion Pharmaceuticals, Marlboro, MA, USA \\ ${ }^{2}$ Evidera, Lexington, $M A, U S A$
}

\begin{abstract}
Objective: To estimate the economic burden of hyperglycemia and diabetes associated with lurasidone versus other atypical antipsychotics in schizophrenia.

Methods: A discrete event simulation model was developed to conduct the analysis from both US payer and societal perspectives over a 3-year timeframe. The model, based on the values of metabolic parameters at 1 year of treatment, identified patients with hyperglycemia at that point and predicted incident cases of diabetes over 3 years using a risk equation from the Framingham Heart Study. Direct and indirect costs of diabetes and hyperglycemia were estimated, according to patients' disease status over time. Data on metabolic changes at 1 year were mainly from 4 clinical studies of lurasidone, supplemented with published data for other atypical antipsychotics. Cost inputs (2012 US dollars) were obtained from published sources.

Results: Compared with olanzapine, risperidone, and quetiapine XR, lurasidone was predicted to result in 2,785,351, and 847 fewer hyperglycemia cases and 1,142,153, and 67 fewer incident diabetes cases per 10,000 patients over 3 years, respectively. Consequently, there was a reduction in the economic burden of diabetes and hyperglycemia by approximately \$23 million, \$2.9 million, and \$3.4 million from a US payer perspective, and \$28 million, \$3.6 million, and $\$ 3.7$ million from a societal perspective.

Conclusion: Adverse metabolic effects of atypical antipsychotics could have substantial unfavorable clinical and economic consequences. Lurasidone may reduce these burdens and may be a treatment alternative for patients with schizophrenia.
\end{abstract}

Keywords: Atypical antipsychotics, discrete event simulation, economic evaluation, lurasidone, metabolic effect, schizophrenia.

\section{INTRODUCTION}

Around 1 in every 100 adults in the United States (US) has schizophrenia [1], a chronic and disabling neurodegenerative disorder characterized by a variable mixture of 'positive' symptoms (e.g., hallucinations, delusions, disordered thinking), 'negative' symptoms (e.g., decreased motivation, social withdrawal, self-neglect), and cognitive deficits [2]. The course of schizophrenia is also variable and often cyclical, with some patients experiencing acute relapses followed by periods of stability [2]. Others, however, remain chronically ill or slowly worsen over time. This diverse clinical picture is one reason why, despite scientific and therapeutic advances, the management of schizophrenia remains especially challenging, with other factors being; the heterogeneity of clinical response, the adverse effects of treatment, and the high morbidity and mortality associated with the condition $[3,4]$. Predictably, therefore, the economic burden of schizophrenia is substantial for patients, their caregivers, and society. In 2002, for example, the total annual cost of schizophrenia in

*Address correspondence to this author at the Evidera, 430 Bedford St., Suite 300, Lexington, MA 02420, USA; Tel: (781) 960-0229;

Fax: (781) 761-0147; E-mail: shien.guo@evidera.com the US was estimated to be $\$ 62.7$ billion, with indirect costs of 32.4 billion, higher than that of the general populations [5].

A key contributor to such costs is the disease burden from associated metabolic disorders, especially diabetes, which is very common among people with schizophrenia [6]. While the exact contribution of diabetes to the total costs of schizophrenia is unclear, a US survey showed that the annual healthcare spending for individuals with schizophrenia was about $90 \%$ higher for those with diabetes than those without any comorbidity [6]. Additionally, these comorbidities may be attributable not only to schizophrenia itself but also to its treatment $[7,8]$. In particular, atypical antipsychotic drugs, the standard pharmacotherapy for schizophrenia, are associated with adverse metabolic changes that increase the likelihood of diabetes [9-11]. For example, results from two large non-industry-sponsored studies (Clinical Antipsychotic Trials in Intervention Effectiveness [CATIE] and Cost Utility of the Latest Antipsychotic Drugs in Schizophrenia Study [CUtLASS]) demonstrated that certain atypical antipsychotics, particularly olanzapine, are associated with considerable weight gain ( $>7$ percent or more of baseline body weight) and diabetes $[12,13]$. All drugs in this class have been shown to produce metabolic changes to varying 
degrees, resulting in a differential metabolic risk profile [9-11]. Thus, these differences in metabolic profile ought to be considered when making treatment choices for individuals with schizophrenia.

These issues focus attention on lurasidone, a new atypical antipsychotic approved by the Food and Drug Administration (FDA) for schizophrenia [14]. This drug has a lack of affinity for histamine $\mathrm{H}_{1}$ and muscarinic $\mathrm{M}_{1}$ receptors, which have been implicated in weight gain-related adverse events; accordingly, it is not expected to have a substantial adverse impact on metabolic parameters $[14,15]$. In keeping with this, clinical studies published to date indicate that lurasidone has efficacy comparable with that of other atypical antipsychotics and a near-neutral effect on weight gain and other metabolic parameters [16, 17]. As such, widespread use of this drug could, in theory, help to prevent incident treatment-related diabetes and substantially reduce the costs associated with this comorbidity, especially in patients with a poor metabolic risk profile and/or those experiencing adverse metabolic effects with other atypical antipsychotics. To explore such possibilities, the aim of this study was to estimate the economic burden of diabetes and hyperglycemia due to metabolic changes with lurasidone versus other, commonly used, atypical antipsychotics for the management of schizophrenia in the US.

\section{METHODS}

\section{Model Structure}

A discrete event simulation (DES) model was developed to conduct this analysis. This modeling technique conceptualizes the course of a disease and its management at the level of individual patients in terms of events that occur over time [18]. The DES methodology was chosen for this analysis due to its capacity to track metabolic changes and occurrence of relevant events at individual patient level, and use them to predict outcomes of interest (i.e., incidence of hyperglycemia, diabetes, and death) over time. The analysis was conducted from the perspectives of both a US payer and society. Olanzapine, risperidone, and quetiapine XR were selected as treatment comparators to be assessed against lurasidone because they are three of the most commonly used atypical antipsychotics, differ with respect to their metabolic risk profiles, and were included as comparators in the clinical studies of lurasidone $[16,17,19,20]$.

Fig. (1) shows a simplified schematic representation of the model structure. Specifically, at the beginning of the simulation, the model created 10,000 patients by random sampling with replacement from a pool of actual patient profiles obtained from participants in two lurasidone clinical studies [17, 19]. Each patient profile was based on a set of baseline patient and metabolic characteristics, including age, gender, weight, fasting glucose (FG), and lipid parameters. Each patient was then cloned three times to generate a total of four identical cohorts of different individuals, with each cohort being assigned to each treatment considered in this analysis (i.e., lurasidone, olanzapine, risperidone, or quetiapine XR). The cloning step prior to treatment assignment resembles perfect randomization, in that the four treatment groups consisted of exactly the same cohort of patients. Cloning patients before allocating the treatment ensures that the only variance between the groups is the assigned treatment.

Following the treatment assignment, the model updated the metabolic parameters for each patient based on metabolic changes at 1 year associated with the allocated treatment, and used the updated values to identify the following groups: i) patients with diabetes, defined as FG $>126 \mathrm{mg} / \mathrm{dL}$ and

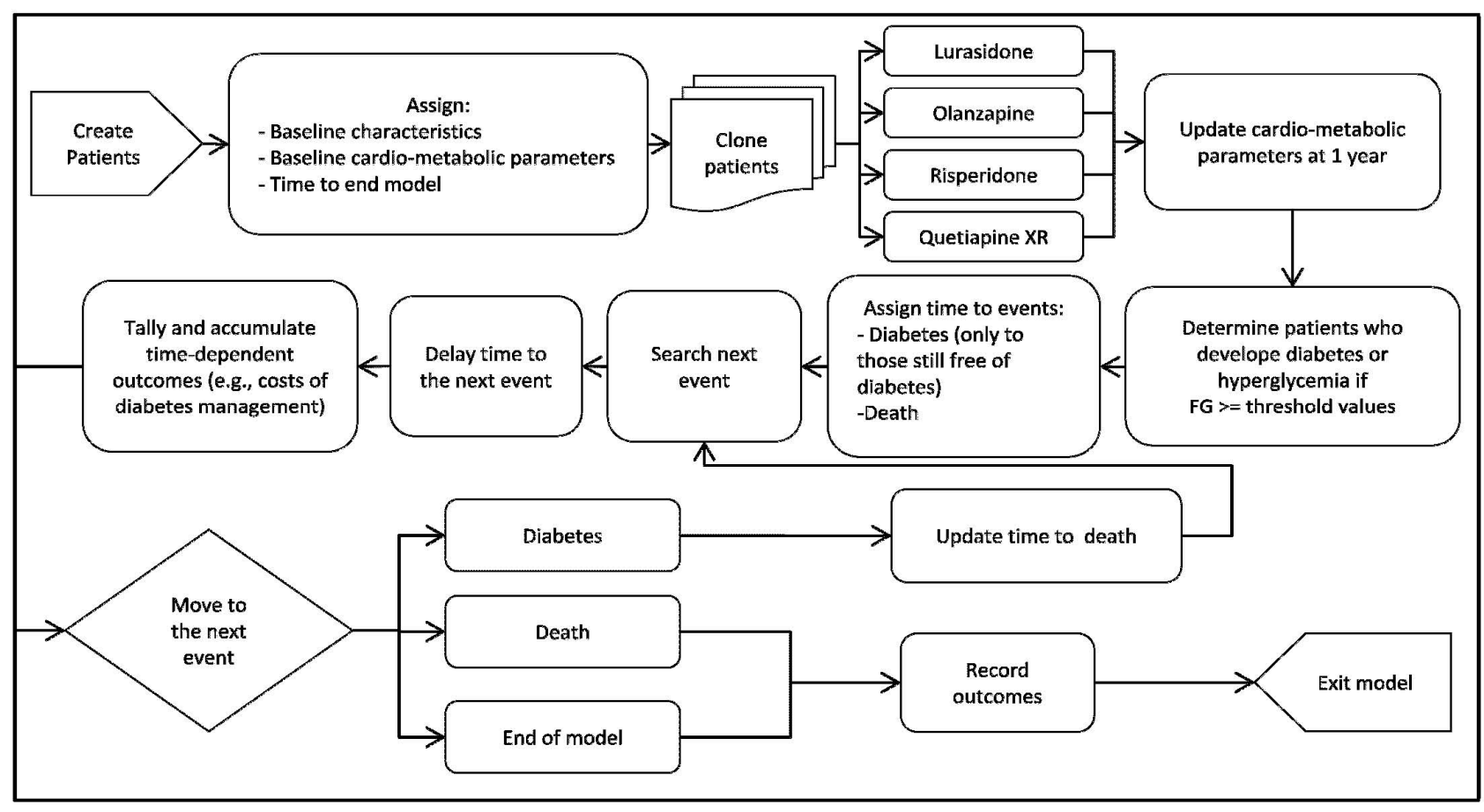

Fig. (1). Schematic representation of the model. FG = fasting glucose. 
ii) patients with hyperglycemia (i.e., pre-diabetes), defined as FG between $100-126 \mathrm{mg} / \mathrm{dL}$. These threshold values were based on recommendations in clinical guidelines [21]. For those patients still free of diabetes at the end of 1 year of treatment, the time for the development of diabetes was predicted using a diabetes risk equation derived from the Framingham Heart Study (FHS) [22]. The model then searched for the next event (i.e., diabetes, death, or end of the model time) for each patient using the shortest time of occurrence and processed the consequences associated with the event. If the next event was diabetes, the patient's risk of death was adjusted and the disease status was updated. Timedependent outcomes were accrued as time elapsed in the model, according to patient's disease status over this duration. These outcomes included costs of clinical management for diabetes and hyperglycemia, as well as costs of absenteeism and presenteeism associated with diabetes. The model simulated patients from one event to another until the model time ended or they died, whichever occurred first.

\section{Model Parameters and Data Sources}

\section{Model Settings}

The base case analyses were simulated over 3 years based on 10,000 patients per treatment group for a total of 100 replications. The 3-year model timeframe was chosen because predictions beyond it are less likely to be supported by the 1-year trial data, and 3 years appears in keeping with many US payers' views of a realistic time horizon for budgetary planning purposes. Costs were discounted at 3\% per annum, in accordance with the recommendations of the US Panel on Cost-Effectiveness in Health and Medicine [23].

\section{Patient Characteristics at Baseline}

Patient profiles $(\mathrm{n}=967)$ obtained from participants in two of the lurasidone clinical studies (i.e., studies PEARL 1 and PEARL 2) $[17,19]$ were used to create the simulated individuals. These patients had a mean age of 38 years, $74 \%$ were male, and $33.7 \%$ were black. Average baseline FG was $95.2(\mathrm{SD}=20.2) \mathrm{mg} / \mathrm{dL}$, high-density lipoprotein (HDL) concentration $47.7(\mathrm{SD}=13.1) \mathrm{mg} / \mathrm{dL}$, weight 77.2 $(\mathrm{SD}=19.5) \mathrm{kg}$, and body mass index (BMI) $26.3(\mathrm{SD}=5.7)$ $\mathrm{kg} / \mathrm{m}^{2}$. Data on parental history of diabetes were not collected in the lurasidone clinical studies, and therefore a prevalence of $27.0 \%$, derived from published literature, was used to impute this characteristic [24].

\section{Treatment Effect on Metabolic Parameters}

Table 1 shows the mean metabolic changes at 1 year by treatment. For lurasidone, the data were obtained by pooling the metabolic effects observed from four of the lurasidone clinical studies $[16,20,25,26]$. For risperidone and quetiapine XR, these inputs were drawn from two 12-month, parallel group, comparative studies of lurasidone $[16,20]$. Because olanzapine patients crossed over to lurasidone after 6 weeks in the lurasidone study NCT00615433 [17, 25], 1-year olanzapine data were obtained from published literature [27]. Changes in BMI were estimated in the model using patients' weight change and height. The model assumed that the values of the metabolic parameters at 1 year of treatment stayed constant over time, and that the metabolic changes at 1 year were the same for all patients within a given treatment group, regardless of their baseline characteristics.

\section{Clinical Events}

\section{Incident Diabetes}

For patients still free of diabetes after 1 year of treatment, the model predicted their time to development of diabetes based on a logistic regression equation derived from the FHS [22]. This risk equation uses BMI, triglyceride concentration, HDL concentration, and FG, as well as other demographic factors, to predict the likelihood of developing diabetes over a 7-year timeframe. The model assumed an exponential distribution for the risk of diabetes when estimating the time to development of the condition based on the risk equation.

\section{Mortality}

The background mortality for individuals with schizophrenia was estimated based on patients' gender and age. Gender-specific mortality data for the general population from the 2006 US Life Table [28] were used and fitted to a Gompertz distribution (Table 1). The risk of death for the general population was then inflated based on published standardized mortality ratios [29], in order to reflect the higher mortality of patients with schizophrenia. For those individuals who developed diabetes, the risk of death was elevated by a factor of 1.76 for males and by 1.92 for females, based on published data (Table 1) [30].

\section{Costs}

The analysis was conducted from both US payer and societal perspectives. The former considered the direct medical costs associated with the management of hyperglycemia and diabetes [31,32], and the latter included, in addition, the costs of absenteeism and presenteeism due to diabetes. All cost inputs were obtained from published literature (Table 1). Specifically, the direct medical costs of managing diabetes and the indirect costs of absenteeism and presenteeism due to diabetes were obtained from the American Diabetes Association (ADA) [31]. The direct costs of managing hyperglycemia were based on the cost estimate from a large cohort of individuals with pre-diabetes enrolled in a health maintenance organization (HMO) plan [32]. All costs in this analysis are reported in 2012 US dollars.

\section{Analyses}

Model outcomes for the base case analysis included: (i) number of patients experiencing hyperglycemia at after the end of 1 year of treatment, (ii) incident cases of diabetes and death over 3 years, and (iii) average direct and indirect costs associated with diabetes and hyperglycemia over 3 years.

One-way sensitivity analyses were conducted to evaluate how the model outcomes varied in relation to changes in key model parameters. Also, in order to account for statistical uncertainties of multiple key parameters, probabilistic sensitivity analyses (PSA) were performed by simultaneously varying the following parameters: mean metabolic changes at 1 year; hazard ratios of death for diabetes; and costs of managing diabetes and hyperglycemia. To measure the uncertainty around a specific parameter, the analysis used the standard error available for that parameter 
Table 1. Model parameters.

\begin{tabular}{|c|c|c|c|c|}
\hline Active Treatment Effects, Mean (SE) & Lurasidone & Olanzapine & Risperidone & Quetiapine XR \\
\hline $\mathrm{HDL}(\mathrm{mg} / \mathrm{dL})$ & $-1.05(0.13)$ & $-3.00(0.38)$ & $-2.60(0.33)$ & $5.20(0.66)$ \\
\hline Triglycerides (mg/dL) & $-9.47(1.21)$ & $30.10(3.84)$ & $15.10(1.93)$ & $-11.40(1.45)$ \\
\hline Parameters for background mortality & Male & Female & & \\
\hline Lambda $(\lambda)$ & -8.738 & -9.468 & & \\
\hline $\operatorname{Gamma}(\gamma)$ & 0.075 & 0.080 & & \\
\hline Hazard ratio of death for DM (SE) & $1.76(0.011)$ & $1.91(0.009)$ & & \\
\hline \multirow[t]{2}{*}{ Indirect Costs Associated with Diabetes by Age (Per Year) } & \multicolumn{2}{|c|}{ Absenteeism } & \multicolumn{2}{|c|}{ Presenteeism } \\
\hline & Male & Female & Male & Female \\
\hline $18-34$ years & $\$ 26$ & $\$ 58$ & $\$ 1,703$ & $\$ 986$ \\
\hline $35-44$ years & $\$ 116$ & $\$ 55$ & $\$ 3,367$ & $\$ 1,610$ \\
\hline $45-54$ years & $\$ 576$ & $\$ 280$ & $\$ 3,149$ & $\$ 1,530$ \\
\hline $55-59$ years & $\$ 420$ & $\$ 209$ & $\$ 2,565$ & $\$ 1,277$ \\
\hline $60-64$ years & $\$ 211$ & $\$ 84$ & $\$ 1,772$ & $\$ 705$ \\
\hline
\end{tabular}

$\mathrm{FG}=$ fasting glucose, $\mathrm{HDL}=$ high-density lipoprotein, $\mathrm{DM}=$ diabetes mellitus, $\mathrm{SE}=$ standard error, $\mathrm{XR}=$ extended-release $, \mathrm{mg}=\mathrm{milligram}, \mathrm{dL}=$ decilitre $\mathrm{kg}=\mathrm{kilogram}$.

from its source data, wherever possible. However, where a

standard error was not available for a selected parameter, $25 \%$ of the mean was used as an assumed standard error-a common approach used in economic models [33, 34]. In general, the model assumed normal distribution for all the parameters when sampling the parameter values in the PSA, the one exception being the hazard ratios of death for diabetes, for which lognormal distribution was assumed.

\section{RESULTS}

\section{Base Case}

Compared with olanzapine, risperidone, and quetiapine $\mathrm{XR}$, lurasidone was predicted to yield the lowest incidence of hyperglycemia (Table 2), with 2,785,351, and 847 fewer cases per 10,000 patients at the end of 1 year of treatment, respectively. The base case results also indicated that treatment with lurasidone would result in the fewest number of new diabetes cases over 3 years. Per 10,000 patients, lurasidone was predicted to have 1,142,153, and 67 fewer incident diabetes cases when compared with olanzapine, risperidone, quetiapine $\mathrm{XR}$, respectively. Treatment with lurasidone also yielded the lowest total number of deaths over 3 years, although the differences between treatments were small (Table 2).
Consequently, lurasidone was predicted to reduce the economic burden of hyperglycemia and diabetes, from a US payer perspective, by an average of: i) $\$ 2,296$ per patient over 3 years when compared with olanzapine; (ii) \$293 per patient when compared with risperidone; and (iii) $\$ 338$ per patient when compared with quetiapine XR. From a societal perspective (i.e., including the costs of productivity and absenteeism due to incident diabetes), lurasidone was predicted to lower the economic burden over 3 years by $\$ 2,821$ per patient versus olanzapine; $\$ 360$ per patient versus risperidone; and $\$ 372$ per patient versus quetiapine XR. Assuming that 10,000 patients with schizophrenia treated with lurasidone for a year, a total expected savings in direct medical costs associated with managing hyperglycemia and diabetes would be approximately $\$ 23$ million compared with olanzapine; \$2.9 million compared with risperidone; and \$3.4 million compared with quetiapine XR from a payer perspective over a 3-year interval. From a societal perspective, the savings could amount to $\$ 28$ million, 3.6 million, and 3.7 million per 10,000 patients, compared with olanzapine, risperidone, and quetiapine XR, respectively.

\section{Sensitivity Analysis}

\section{Deterministic Sensitivity Analyses}

One-way sensitivity analyses were also carried out to evaluate how sensitive the estimated net savings were to 
Table 2. Base-case results.

\begin{tabular}{|c|c|c|c|c|}
\hline Outcome & Lurasidone & Olanzapine & Risperidone & Quetiapine XR \\
\hline \multicolumn{5}{|c|}{ Clinical Events (per 10,000 patients over 3 years) } \\
\hline Hyperglycemia & 2,921 & 5,706 & 3,272 & 3,768 \\
\hline Diabetes & 2,766 & 3,908 & 2,919 & 2,833 \\
\hline Deaths & 64 & 76 & 66 & 64 \\
\hline \multicolumn{5}{|c|}{ Costs (discounted, per patient over 3 years) } \\
\hline \multicolumn{5}{|l|}{ Direct Costs } \\
\hline Hyperglycemia & $\$ 698$ & $\$ 1,338$ & $\$ 780$ & $\$ 926$ \\
\hline Diabetes & $\$ 4,477$ & $\$ 6,133$ & $\$ 4,688$ & $\$ 4,587$ \\
\hline TOTAL DIRECT COSTS & $\$ 5,175$ & $\$ 7,471$ & $\$ 5,468$ & $\$ 5,513$ \\
\hline \multicolumn{5}{|l|}{ Indirect Costs } \\
\hline Absenteeism & $\$ 133$ & $\$ 175$ & $\$ 138$ & $\$ 135$ \\
\hline Presenteeism & $\$ 1,371$ & $\$ 1,854$ & $\$ 1,433$ & $\$ 1,403$ \\
\hline TOTAL INDIRECT COSTS & $\$ 1,504$ & $\$ 2,029$ & $\$ 1,571$ & $\$ 1,538$ \\
\hline TOTAL COST & $\$ 6,679$ & $\$ 9,500$ & $\$ 7,039$ & $\$ 7,051$ \\
\hline
\end{tabular}

$\mathrm{XR}=$ extended-release.

changes in each model parameter. Results of these analyses showed that the net savings were most sensitive to changes in model time horizon, metabolic effect of treatment, and annual cost of managing diabetes (Figs. 2-4), and least sensitive to changes in hazard ratios of death for diabetes.

\section{Probabilistic Sensitivity Analyses (PSA)}

From a US payer perspective, the PSA showed that lurasidone was a cost-saving therapeutic option in: i) $100 \%$ of the 1,000 replications, with a range of cost reduction from $\$ 835$ to $\$ 5,494$ per patient over 3 years, when compared with olanzapine; ii) $94 \%$ of the replications, with a range of $-\$ 288$ to $\$ 1,015$ per patient, when compared with risperidone; and iii) $90 \%$ of the replications, with a range of $-\$ 527$ to $\$ 1,103$ per patient, when compared with quetiapine XR. From a societal perspective, the PSA indicated that lurasidone was a cost-saving therapeutic option in $100 \%, 94 \%$, and $92 \%$ of the 1,000 replications, when compared with olanzapine,

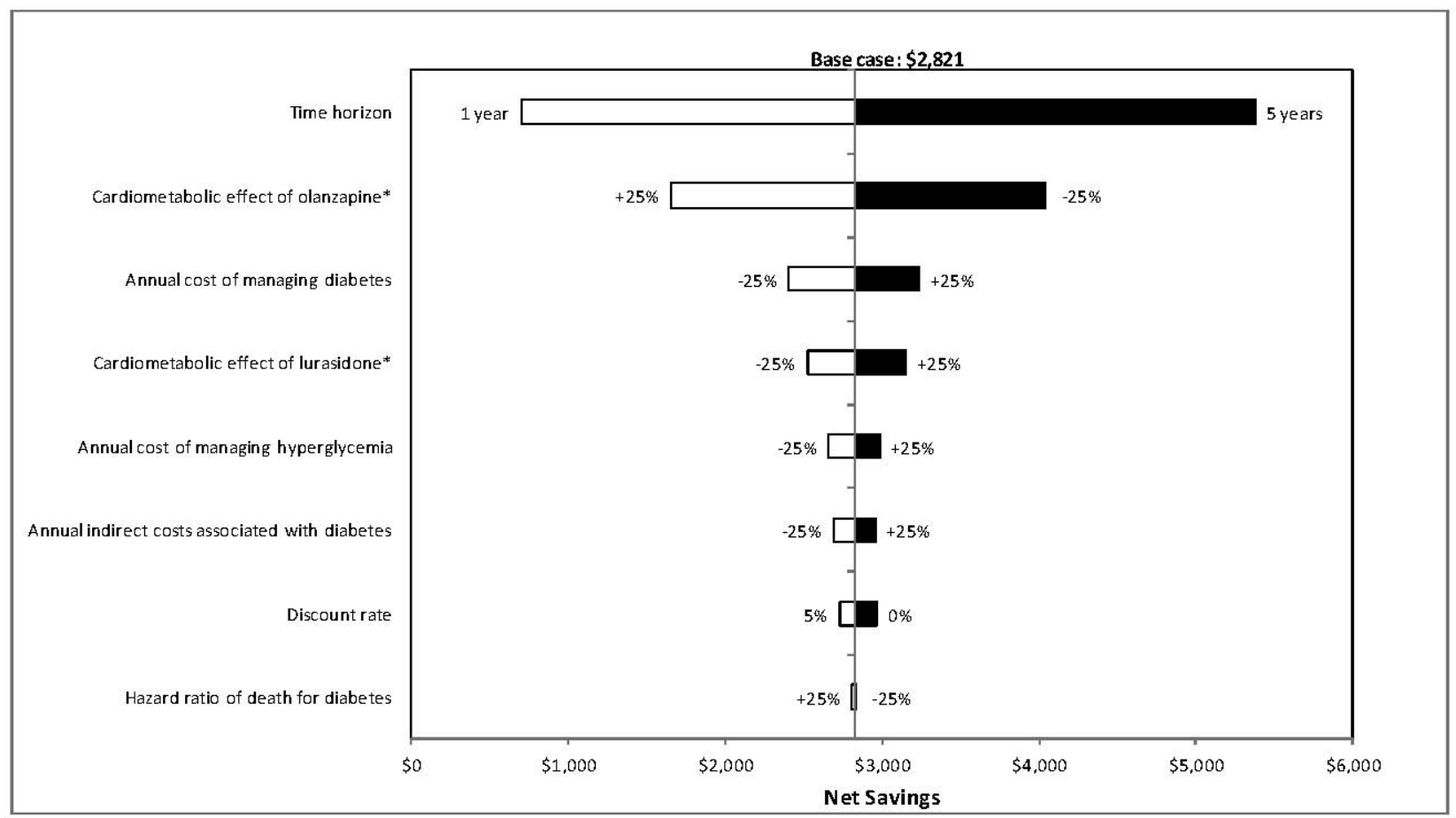

Fig. (2). Results of deterministic sensitivity analyses for lurasidone $v s$ olanzapine (based on direct and indirect costs). *Metabolic effect plus or minus $25 \%$ means that the metabolic effect of a treatment improves or worsens by $25 \%$, respectively. White bars indicate cost savings with lurasidone decrease relative to the base-case. Black bars indicate cost savings with lurasidone increase relative to the base-case. 


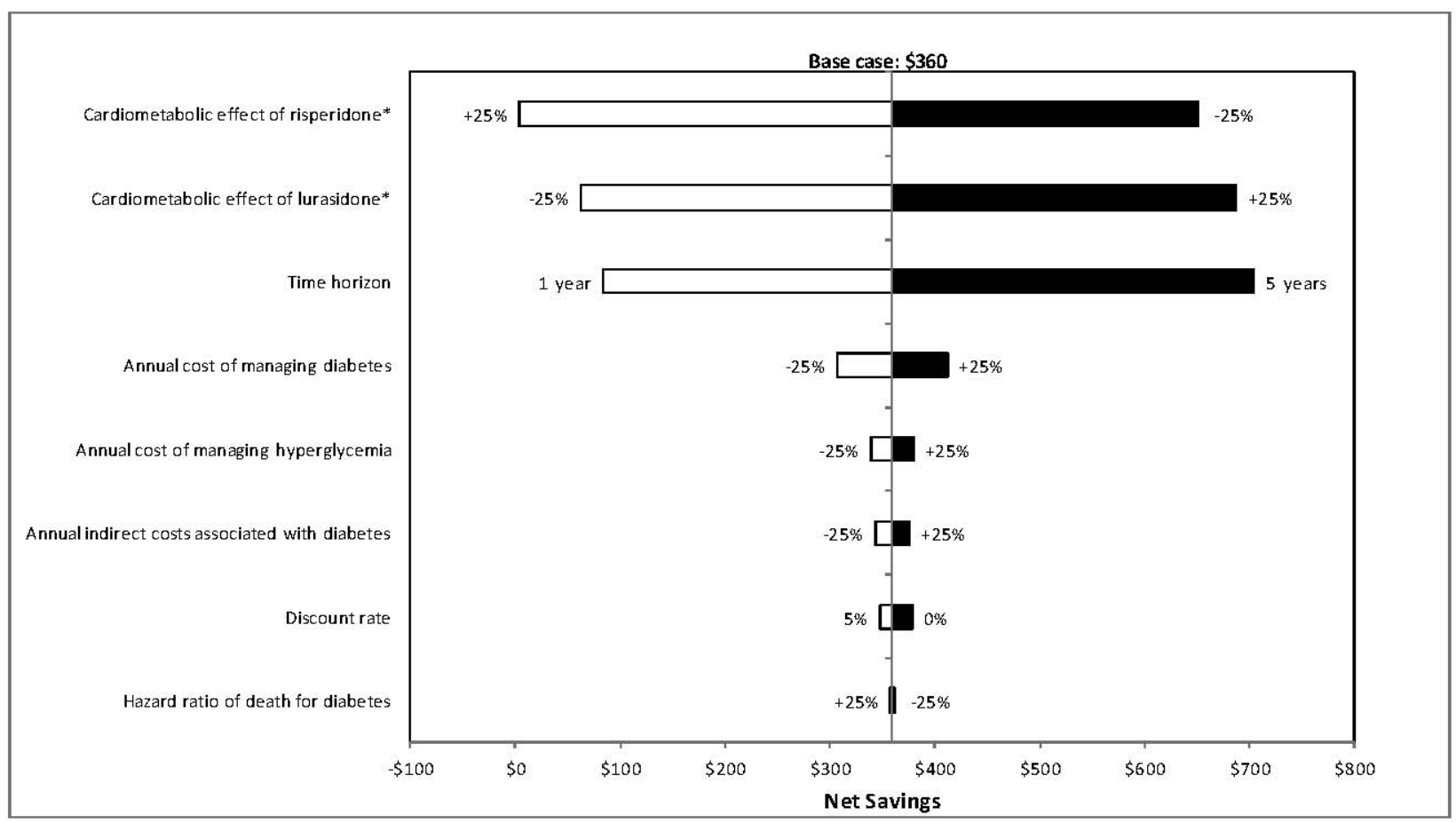

Fig. (3). Results of deterministic sensitivity analyses for lurasidone $v s$ risperidone (based on direct and indirect costs). *Metabolic effect plus or minus $25 \%$ means that the metabolic effect of a treatment improves or worsens by $25 \%$, respectively. White bars indicate cost savings with lurasidone decrease relative to the base-case. Black bars indicate cost savings with lurasidone increase relative to the base-case.

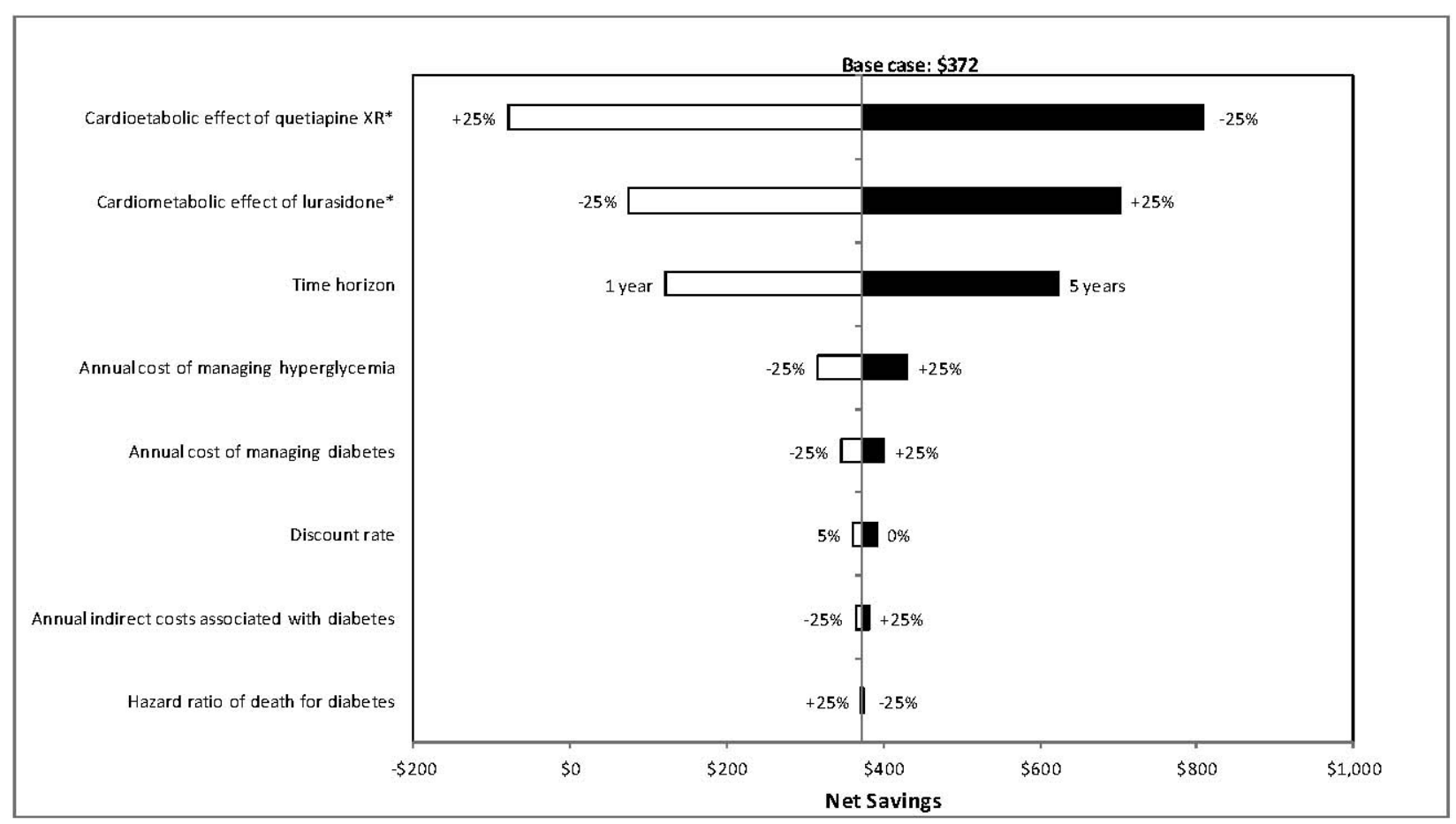

Fig. (4). Results of deterministic sensitivity analyses for lurasidone $v s$ quetiapine $\mathrm{XR}$ (based on direct and indirect costs). XR $=$ extendedrelease. *Metabolic effect plus or minus $25 \%$ means that the metabolic effect of a treatment improves or worsens by $25 \%$, respectively. White bars indicate cost savings with lurasidone decrease relative to the base-case. Black bars indicate cost savings with lurasidone increase relative to the base-case. 
risperidone, and quetiapine, respectively, with a range of cost reduction from $\$ 681$ to $\$ 4,470$ per patient with olanzapine, $-\$ 242$ to $\$ 846$ per patient with risperidone, and -\$405 to $\$ 947$ per patient with quetiapine XR.

\section{DISCUSSION}

This analysis estimated the economic burden of diabetes and hyperglycemia due to metabolic changes associated with 1 -year treatment with lurasidone, olanzapine, risperidone, and quetiapine XR over a 3-year time horizon. The results indicated that lurasidone would result in the fewest new cases of hyperglycemia and diabetes, resulting in the lowest total cost associated with managing these problems. These model outcomes were robust, as indicated by the results of the PSA. The difference in total cost was the greatest when compared with olanzapine, and the least when compared with risperidone. Such findings are consistent with the 2004 consensus guidelines jointly published by the ADA and American Psychiatric Association [35] with respect to the magnitude of underlying risk of diabetes associated with the treatments considered in this analysis.

As indicated in the results of this analysis, the savings from preventing diabetes and hyperglycemia due to treatment with lurasidone could be substantial, especially when compared with olanzapine. The amount of savings with lurasidone from a societal perspective could be greater if other indirect costs, such as unemployment due to diabetes-related disabilities and loss of productivity due to diabetes-related deaths are also considered. Additionally, the model population was relatively young (with a mean age of 38 years), and had a smaller cost of absenteeism and presenteeism per capita, as indicated by the literature, due to fewer days absent per worker per year and lower average daily earnings [31]. The estimated savings could also be higher if older populations (i.e., age 40-60) are considered for inclusion in this analysis.

It should also be noted that this analysis focused only on the economic burden of diabetes and hyperglycemia due to metabolic changes associated with atypical antipsychotic treatments. Other costs, such as costs of drug acquisition, hospitalizations due to relapses, and management of other side effects, were not considered. The amount of savings with lurasidone may increase or decrease if these costs are included in this analysis. This question can be perhaps answered by incorporating the findings from a recent economic analysis published by O'Day and colleagues who performed a cost-effectiveness analysis comparing lurasidone with other commonly used atypical antipsychotics including generic risperidone, generic olanzapine, quetiapine $\mathrm{XR}$, generic ziprasidone, and aripiprazole in the treatment of adults with schizophrenia from a US payer perspective [36]. This study estimated that the total discounted costs (in 2012 US dollars) of antipsychotics and hospitalizations due to relapses associated with lurasidone, generic olanzapine, generic risperidone, and quetiapine XR were $\$ 101,089$, $\$ 101,196, \$ 99,479$, and $\$ 104,290$ per patient over 5 years, respectively. Therefore, based on the study findings from O'Day and colleagues, we would expect that the savings with lurasidone are more likely to increase when compared with generic olanzapine and quetiapine XR if the costs of antipsychotics and hospitalizations due to relapses are considered in our analysis. When compared with generic risperidone, lurasidone would result in a greater overall cost in our analysis, but the cost $(\$ 25,884)$ of relapse-related hospitalizations avoided for lurasidone versus generic risperidone estimated by O'Day and colleagues would be smaller (i.e., lurasidone would be even more cost-effective).

One additional important issue to highlight is that both our and O'Day's analyses did not include the cost of managing other treatment-related side effects such as extrapyramidal symptoms. A recent meta-analysis published by Leucht and colleagues shows that lurasidone incurred a greater risk of extrapyramidal side effects compared with olanzapine and quetiapine [37]. This would potentially reduce the magnitude of the savings with lurasidone if such costs are included, but the potential reduction should be small as the differences in risk of experiencing extrapyramidal symptoms between these treatments were not large. However, in order to provide a complete picture of the economic impact of lurasidone relative to other treatments, the costs of treating other side effects should be considered in future economic studies of lurasidone.

The deterministic sensitivity analyses indicated that the net savings with lurasidone were mostly influenced by the time horizon of the model. A longer horizon would yield greater cost savings with lurasidone, as more diabetes events could be avoided and the economic burden associated with diabetes could be lessened. However, this analysis used a conservative 3-year model time horizon for the projection of findings based on the data from 1-year studies. The net savings with lurasidone could also be greatly influenced by the metabolic effects associated with treatments. Nevertheless, such an uncertainty to the predicted savings was reduced by the results of the PSA in which the uncertainties with respect to the metabolic effects of treatments were taken into account.

This is the first modeling study to assess the economic burden of diabetes and hyperglycemia due to metabolic changes with lurasidone versus olanzapine, risperidone, and quetiapine $\mathrm{XR}$ in the management of schizophrenia. Its strengths include the use of an individual time-to-event simulation modeling approach that accounts for the variability in baseline patient and disease characteristics that influence the risk of diabetes [38], and the use of comparative clinical data from the same clinical trials (except for olanzapine) to conduct the assessment. However, the analysis also has various limitations. First, the metabolic treatment effect (i.e., changes from baseline to 1 year) was assumed to be the same for all patients on a given medication. This cohort-level assumption might not fully capture the effect of treatment on metabolic parameters and associated diseases, at an individual-patient level. The impact of atypical antipsychotics on metabolic outcomes might be characterized more precisely by future microsimulation analyses that employ predictive equations at a patient level to estimate changes in metabolic parameters over time, based on the treatment received and relevant baseline patient and disease characteristics. Second, the model assumed that the values of metabolic parameters remained constant beyond 1 year of treatment. This assumption may underestimate the potential savings with lurasidone as patients' metabolic profiles tend to get worse 
as they stay longer on those drugs with adverse metabolic effects, such as olanzapine [39]. Third, the model assumed no risk of treatment discontinuation, which apparently does not reflect the real-world practice. However, the impact of this assumption on the predicted outcomes should not be significant as the timeframe of this analysis is short. Even if this is considered, lurasidone should be likely to result in cost savings as indicated by the findings from a study done by O'Day and colleagues [36]. Finally, this analysis considered solely the impact of metabolic effects of atypical antipsychotics. The economic consequence of each treatment may differ if other treatment-related adverse effects are considered. To provide a more balanced view regarding the economic impact of lurasidone versus other antipsychotics, these costs should be considered in future cost-effectiveness analyses of lurasidone in adults with schizophrenia.

The findings from this analysis have policy implications for many state Medicaid and Managed Medicaid programs in which generic olanzapine and risperidone are regarded as first-line treatments for patients with schizophrenia. This analysis suggests that such a policy may result in more costs incurred for managing adverse metabolic events associated with these treatments as patients are less likely to receive the optimal treatment they need due to the limited treatment options. As patients often respond optimally in terms of efficacy and tolerability to a specific treatment but not to others, and drugs within this class are not considered to be interchangeable [40], thus a policy allowing physicians to select a treatment for each individual patient based on his or her existing medical conditions and past treatment history without inflexible restrictions of coverage could potentially lead to cost savings due to improved clinical outcomes.

\section{CONCLUSION}

This analysis indicates that treatment with lurasidone may result in fewer cases of hyperglycemia and diabetes and consequently reduce the economic burden associated with these events, when compared with olanzapine, risperidone and quetiapine XR. Given that clinical response to atypical antipsychotics is heterogeneous among adults with schizophrenia and each drug has a unique efficacy and metabolic profile, lurasidone would be a valuable addition to the therapeutic armamentarium for health care professionals, payers, and patients. Finally, with the growing prevalence of metabolic disorders among adults with schizophrenia at the time of diagnosis or after treatment initiation, its contribution would be increasingly important and greater if it can be widely accessible to patients.

\section{CONFLICT OF INTEREST}

This study was funded by Sunovion Pharmaceuticals Inc., the maker of the products was potentially affected by this work.

\section{ACKNOWLEDGEMENTS}

All authors participated in the conception and implementation of the model, data analyses for obtaining model inputs and analyses of the simulation, and writing of the paper. Shien Guo and Luis Hernandez are employees of Evidera (formerly a division of United BioSource Corporation), a consultancy that has also received grants for other unrelated research from various pharmaceutical companies. Julia Green was an employee of United BioSource Corporation at the time this study was conducted. Krithika Rajagopalan and Antony Loebel are employees of Sunovion Pharmaceuticals, Inc.

\section{DISCLOSURE}

Evidera (formerly a division of United BioSource Corporation) received funding from Sunovion Pharmaceuticals Inc to conduct this study.

\section{REFERENCES}

[1] Narrow WE, Rae DS, Robins LN, Regier DA. Revised prevalence estimates of mental disorders in the United States: using a clinical significance criterion to reconcile 2 surveys' estimates. Arch Gen Psychiatry 2002; 59: 115-23

[2] American Psychiatric Association. Diagnostic and Statistical Manual of Mental Disorders. DSM-IV-TR. 4th ed. Arlington, VA: American Psychiatric Association 2000.

[3] Lehman AF, Lieberman JA, Dixon LB, et al. Practice guideline for the treatment of patients with schizophrenia, $2^{\text {nd }}$ ed. Am J Psychiatry 2004; 161: 1-56.

[4] Tandon R, Belmaker RH, Gattaz WF, et al. World Psychiatric Association Pharmacopsychiatry Section statement on comparative effectiveness of antipsychotics in the treatment of schizophrenia. Schizophr Res 2008; 100: 20-38.

[5] Wu EQ, Birnbaum HG, Shi L, et al. The economic burden of schizophrenia in the United States in 2002. J Clin Psychiatry 2005; 66: 1122-9.

[6] McDermott S, Moran R, Platt T, et al. Heart disease, schizophrenia, and affective psychoses: epidemiology of risk in primary care. Community Ment Health J 2005; 41: 747-55.

[7] Goff DC, Sullivan LM, McEvoy JP, et al. A comparison of tenyear cardiac risk estimates in schizophrenia patients from the CATIE study and matched controls. Schizophr Res 2005; 80: $45-$ 53.

[8] McEvoy JP, Meyer JM, Goff DC, et al. Prevalence of the metabolic syndrome in patients with schizophrenia: baseline results from the Clinical Antipsychotic Trials of Intervention Effectiveness (CATIE) schizophrenia trial and comparison with national estimates from NHANES III. Schizophr Res 2005; 80: 19-32.

[9] Correll CU, Frederickson AM, Kane JM, Manu P. Metabolic syndrome and the risk of coronary heart disease in 367 patients treated with second-generation antipsychotic drugs. J Clin Psychiatry 2006; 67: 575-83.

[10] L'Italien GJ, Casey DE, Kan HJ, Carson WH, Marcus RN Comparison of metabolic syndrome incidence among schizophrenia patients treated with aripiprazole versus olanzapine or placebo. J Clin Psychiatry 2007; 68: 1510-6.

[11] Sernyak MJ, Leslie DL, Alarcon RD, Losonczy MF, Rosenheck R. Association of diabetes mellitus with use of atypical neuroleptics in the treatment of schizophrenia. Am J Psychiatry 2002; 159: 561-6.

[12] Jones PB, Barnes TR, Davies L, et al. Randomized controlled trial of the effect on quality of life of second- $v s$ first-generation antipsychotic drugs in schizophrenia: Cost Utility of the Lates Antipsychotic Drugs in Schizophrenia Study (CUtLASS 1). Arch Gen Psychiatry 2006; 63: 1079-87.

[13] Lieberman JA, Stroup TS, McEvoy JP, et al. Effectiveness of antipsychotic drugs in patients with chronic schizophrenia. N Engl J Med 2005; 353: 1209-23.

[14] Sunovion Pharmaceuticals Inc. LATUDA ${ }^{\circledR}$ (lurasidone $\mathrm{HCl}$ ) Package Insert. LATUDA ${ }^{\circledR}$ (Lurasidone HCL) tablet for oral administration. Prescribing information. Initial U.S. Approval: 2010. Package Insert revised 12/2011. Available at: http://www.accessdata.fda.gov/drugsatfda_docs/label/2011/200603 s004s006lbl.pdf 2011

[15] Kim DH, Maneen MJ, Stahl SM. Building a better antipsychotic: receptor targets for the treatment of multiple symptom dimensions of schizophrenia. Neurotherapeutics 2009; 6: 78-85.

[16] Citrome L, Cucchiaro J, Sarma K, et al. Long-term safety and tolerability of lurasidone in schizophrenia: a 12-month, doubleblind, active-controlled study. Int Clin Psychopharmacol 2012; 27: 165-76.

[17] Meltzer HY, Cucchiaro J, Silva R, et al. Lurasidone in the treatment of schizophrenia: a randomized, double-blind, placebo- 
and olanzapine-controlled study. Am J Psychiatry 2011; 168: 95767.

[18] Caro JJ. Pharmacoeconomic analyses using discrete event simulation. Pharmacoeconomics 2005; 23: 323-32.

[19] Nakamura M, Ogasa M, Guarino J, et al. Lurasidone in the treatment of acute schizophrenia: a double-blind, placebocontrolled trial. J Clin Psychiatry 2009; 70: 829-36.

[20] Loebel A, Sarma K, Xu J, et al. Relapse prevention with lurasidone vs quetiapine XR in chronic schizophrenia: results of a 12-month, double-blind study. Poster presented at: American College of Neuropsychopharmacology; December 4-8, 2011; Waikoloa Beach, HI 2011.

[21] American Diabetes Association. How to Tell if You Have Diabetes or Prediabetes Alexandria, VA: American Diabetes Association 2010 [cited: $17^{\text {th }}$ Oct 2010]; Available from: http://www.diabetes. org/diabetes-basics/prevention/pre-diabetes/diagnosis.html

[22] Wilson PW, Meigs JB, Sullivan L, et al. Prediction of incident diabetes mellitus in middle-aged adults: the Framingham Offspring Study. Arch Intern Med 2007; 167: 1068-74.

[23] Lipscome J, Weinstein MC, Torrance GW. Time Preference. In: Gold MR, Siegel JE, Russell LB et al., Eds. Cost-Effectiveness in Health and Medicine. New York: Oxford University Press 1996.

[24] Fernandez-Egea E, Miller B, Bernardo M, Donner T, Kirkpatrick B. Parental history of type 2 diabetes in patients with nonaffective psychosis. Schizophr Res 2008; 98: 302-6.

[25] A phase 3 randomized, placebo- and active comparator controlled, clinical trial to study the safety and efficacy of two doses of lurasidone $\mathrm{HCl}$ in acutely psychotic patients with schizophrenia. ClinicalTrials.gov Identifier: NCT00615433.

[26] A phase 3 randomized, placebo-controlled, clinical trial to study the safety and efficacy of three doses of lurasidone $\mathrm{HCl}$ in acutely psychotic patients with schizophrenia. ClinicalTrials.gov Identifier: NCT00549718.

[27] Chrzanowski WK, Marcus RN, Torbeyns A, Nyilas M, McQuade RD. Effectiveness of long-term aripiprazole therapy in patients with acutely relapsing or chronic, stable schizophrenia: a 52-week, open-label comparison with olanzapine. Psychopharmacology (Berl) 2006; 189: 259-66.

[28] Human Mortality Database. University of California, Berkeley (USA), and Max Planck Institute for Demographic Research (Germany). www.mortality.org [Accessed: September 15, 2010].
[29] Wildgust HJ, Beary M. Are there modifiable risk factors which will reduce the excess mortality in schizophrenia? J Psychopharmacol 2010; 24: 37-50.

[30] Bertoni AG, Kirk JK, Goff DC Jr, Wagenknecht LE. Excess mortality related to diabetes mellitus in elderly Medicare beneficiaries. Ann Epidemiol 2004; 14: 362-7.

[31] American Diabetes Association. Economic costs of diabetes in the U.S. in 2007. Diabetes Care 2008; 31: 596-615.

[32] Nichols GA, Arondekar B, Herman WH. Medical care costs one year after identification of hyperglycemia below the threshold for diabetes. Med Care 2008; 46: 287-92.

[33] Getsios D, Blume S, Ishak KJ, Maclaine GD. Cost effectiveness of donepezil in the treatment of mild to moderate Alzheimer's disease: a UK evaluation using discrete-event simulation. Pharmacoeconomics 2010; 28: 411-27.

[34] Guo S, Hernandez L, Wasiak R, Gaudig M. Modelling the clinica and economic implications of galantamine in the treatment of mildto-moderate Alzheimer's disease in Germany. J Med Econ 2010; 13: 641-54

[35] American Diabetes Association, American Psychiatric Association, American Association of Clinical Endocrinologists, North American Association for the Study of Obesity. Consensus development conference on antipsychotic drugs and obesity and diabetes. Diabetes Care 2004; 27: 596-601.

[36] O'Day K, Rajagopalan K, Meyer K, Pikalov A, Loebel A. Longterm cost-effectiveness of atypical antipsychotics in the treatment of adults with schizophrenia in the US. Clinicoecon Outcomes Res 2013; 5: 459-70

[37] Leucht S, Cipriani A, Spineli L, et al. Comparative efficacy and tolerability of 15 antipsychotic drugs in schizophrenia: a multipletreatments meta-analysis. Lancet 2013; 382: 951-62.

[38] Heeg BM, Damen J, Buskens E, et al. Modelling approaches: the case of schizophrenia. Pharmacoeconomics 2008; 26: 633-48

[39] Newcomer JW, Campos JA, Marcus RN, et al. A multicenter, randomized, double-blind study of the effects of aripiprazole in overweight subjects with schizophrenia or schizoaffective disorder switched from olanzapine. J Clin Psychiatry 2008; 69: 1046-56.

[40] Simpson GM. Atypical antipsychotics and the burden of disease. Am J Manag Care 2005; 11: S235-41.

This is an open access article licensed under the terms of the Creative Commons Attribution Non-Commercial License (http://creativecommons.org/licenses/bync/3.0/) which permits unrestricted, non-commercial use, distribution and reproduction in any medium, provided the work is properly cited. 